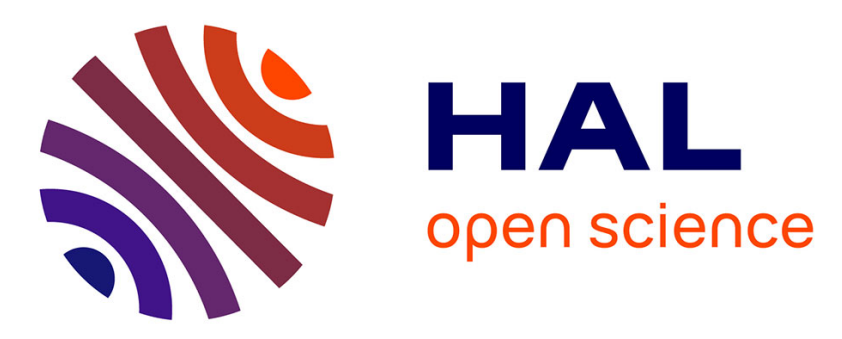

\title{
Métabolisme du fer : impact de l'hypoactivité et mécanismes sous-jacents
}

K. Nay, M. Horeau, Olivier Loréal, F. Derbré

\section{To cite this version:}

K. Nay, M. Horeau, Olivier Loréal, F. Derbré. Métabolisme du fer: impact de l'hypoactivité et mécanismes sous-jacents. Cahiers de Nutrition et de Diététique, 2021, 56 (2), pp.131-140. 10.1016/j.cnd.2021.01.002 . hal-03225511

\section{HAL Id: hal-03225511 \\ https://hal.science/hal-03225511}

Submitted on 20 May 2021

HAL is a multi-disciplinary open access archive for the deposit and dissemination of scientific research documents, whether they are published or not. The documents may come from teaching and research institutions in France or abroad, or from public or private research centers.
L'archive ouverte pluridisciplinaire HAL, est destinée au dépôt et à la diffusion de documents scientifiques de niveau recherche, publiés ou non, émanant des établissements d'enseignement et de recherche français ou étrangers, des laboratoires publics ou privés. 
Métabolisme du fer : impact de l'hypoactivité et mécanismes sous-jacents Iron metabolism: impact of hypoactivity and underlying mechanisms

Kevin Nay ${ }^{1}$, Mathieu Horeau ${ }^{1}$, Olivier Loréal $^{2}$, Frédéric Derbré $^{1 \#}$

${ }^{1}$ Laboratoire "Mouvement, Sport, Santé » (M2S) - EA7470, Université de Rennes/ENS Rennes, France

${ }^{2}$ INSERM, Université de Rennes, INRAE, UMR 1241, Plate-forme AEM2, Unité "Nutrition, Métabolismes et Cancer » (NuMeCan), Rennes, France

\section{${ }^{\#}$ Correspondant:}

Fréderic Derbré, PhD, Maitre de conférences

Laboratoire "Mouvement, Sport, Santé" - EA7470

Université de Rennes / ENS Rennes, 35170 Bruz, France

Mail : frederic.derbre@univ-rennes2.fr 
Résumé : En situation d'impesanteur ou d'alitement, astronautes et patients hospitalisés subissent une réduction considérable de leur activité musculaire, qualifiée d'hypoactivité, qui impacte directement leur état de santé. En raison de son implication dans le transport et le stockage tissulaire de l'oxygène tout comme dans le métabolisme énergétique, le fer et son métabolisme pourraient jouer un rôle essentiel dans certaines des altérations physiologiques associées à l'hypoactivité. Sa redistribution de manière anormale pourrait en effet contribuer à l'anémie et le stress oxydant hautement délétère observé dans certains organes en situation d'hypoactivité (i.e. os et muscle squelettique). Dans ce contexte, l'objectif de cette revue de synthèse est de présenter les connaissances actuelles concernant la régulation du métabolisme du fer en réponse à l'hypoactivité, et d'ouvrir des pistes de réflexion pour améliorer la prise en charge nutritionnelle des astronautes et des patients alités.

Mots-clés : inactivité physique, microgravité, hepcidine, inflammation, déconditionnement

\section{Points essentiels}

- L'hypoactivité définit une très faible activité musculaire sur une durée prolongée

- L'hypoactivité s'observe principalement chez les astronautes et les patients alités

- L'hypoactivité provoque une redistribution du fer dans l'organisme

- L'hepcidine joue un rôle clé dans cette redistribution

- Cette redistribution pourrait impacter les capacités fonctionnelles à long terme 


\begin{abstract}
Under microgravity or during bedrest, astronauts and patients exhibit a major reduction in muscle activity - also called hypoactivity - that directly affects health status. To improve astronauts' health during and after missions, or bedridden patients' care, identifying the underlying mechanisms is necessary. Since it seems to interact with skeletal muscle, iron metabolism could play a key role. Iron is indeed critical to maintain the homeostasis of the human body through its essential role in energy metabolism, cellular respiration and oxygen transport. Under hypoactivity, iron misdistribution could impact the organism by promoting anemia and oxidative stress which are highly deleterious for some organs. In this review, we describe the current knowledge about the regulation of iron metabolism under hypoactivity and propose new ways to improve nutritional care of astronauts and bedridden patients.
\end{abstract}

Keywords: physical inactivity, microgravity, hepcidin, inflammation, unloading 


\section{Introduction}

Durant ces dix dernières années, l'effervescence autour de la conquête spatiale s'est mondialement intensifiée. Après l'annonce du retour de Thomas Pesquet à bord de la station spatiale internationale, fin 2020, et l'intérêt que porte la Chine pour la face cachée de la Lune, de l'autre côté du pacifique, c'est la NASA qui a annoncé son envie d'envoyer le premier Homme sur Mars d'ici 2033. À la veille de telles expéditions, les agences spatiales internationales ont multiplié les études sur les astronautes dans le but de préserver au mieux leur santé. L'hostilité de l'environnement spatial est notamment caractérisée par l'absence d'attraction gravitationnelle réduisant ainsi considérablement l'activité musculaire de l'astronaute. Le terme d'hypoactivité est utilisé pour caractériser la très faible activité d'un ou plusieurs groupes musculaires pendant une période plus ou moins longue. Cette hypoactivité impacte également les patients en réanimation qui demeurent alités pendant plusieurs jours ou semaines. Dans ce contexte, l'extrême diminution des contraintes mécaniques qui s'exerce sur le système musculo-squelettique ainsi que les altérations cardio-vasculaires associées vont aggraver l'état de santé de l'astronaute ou du patient [1-3]. Ces altérations vont impacter les capacités fonctionnelles de l'astronaute limitant sa capacité à conduire les missions qui lui sont confiées (Coker et al. 2015; Moore et al. 2014). Pour le patient alité, la réduction de ses capacités fonctionnelles conditionnera directement la durée de sa convalescence, voir même son retour à une vie autonome et indépendante si ce patient est âgé.

Lors des 15 dernières années, des études conduites par la NASA et nos équipes de recherche se sont intéressées au métabolisme du fer en réponse à l'hypoactivité mettant notamment en évidence des anomalies de sa distribution dans l'organisme. Le fer, en jouant un rôle essentiel dans le métabolisme énergétique, la respiration cellulaire et surtout dans le transport de l'oxygène aux différents tissus, est indispensable au maintien de l'homéostasie de différents métabolismes de notre organisme. Sa redistribution de manière anormale pourrait jouer un rôle dans la réduction des capacités fonctionnelles des astronautes et des patients alités en favorisant une anémie et un stress oxydant hautement délétère pour les organes. La compréhension des mécanismes sous-jacents constitue donc un enjeu important pour améliorer la prise en charge nutritionnelle des astronautes, mais également celle des patients alités. Dans ce contexte, cette revue a pour ambition de présenter un état des lieux des liens existants entre le métabolisme du fer et l'hypoactivité. 


\section{Généralités sur le métabolisme du fer et l'hepcidine, son chef d'orchestre}

Le fer est présent principalement dans l'organisme sous deux formes ioniques : le fer ferrique $\left(\mathrm{Fe}^{3+}\right)$, insoluble, et le fer ferreux $\left(\mathrm{Fe}^{2+}\right)$ qui est lui soluble. Le passage d'un état à l'autre lui permet ainsi d'accepter ou de donner des électrons lui conférant un rôle clé dans de nombreuses réactions d'oxydo-réduction [6]. Le fer est indispensable à l'homéostasie de notre organisme en étant un cofacteur essentiel de diverses enzymes impliquées dans des réactions métaboliques essentielles à la production d'énergie par la chaine respiratoire mitochondriale ou à la synthèse de l'ADN. Le fer, complexé à une proto-porphyrine, forme un hème qui est le cofacteur central de l'hémoglobine et de la myoglobine, permettant respectivement d'approvisionner les différents organes en oxygène et de le stocker au niveau musculaire. En effet, c'est au fer de l'hème que se fixe de façon réversible le dioxygène dans ces deux protéines. Pour ces raisons, une carence en fer est responsable d'un grand nombre de dysfonctionnements métaboliques. Toutefois, le fer sous forme ferreux $\left(\mathrm{Fe}^{2+}\right)$ est très instable et réactif. Il est à l'origine de la formation d'espèces réactives dérivés de l'oxygène (ERDO) via la réaction de Fenton $\left(\mathrm{Fe}^{2+}+\right.$ $\mathrm{H}_{2} \mathrm{O}_{2} \rightarrow \mathrm{Fe}^{3+}+\mathrm{HO}^{\circ}+\mathrm{HO}^{-}$) conduisant à générer le radical hydroxyle, composé très réactif conduisant à des dommages oxydants cellulaires pouvant causer un stress oxydant. Pour éviter la formation excessive de ces ERDO, le stockage et le transport du fer sont étroitement contrôlés au sein de l'organisme et des cellules. Malgré ce contrôle, une concentration en fer anormale peut apparaitre et entrainer un stress oxydant en cas de surcharge, ou une anémie en cas de carence, ce qui peut engendrer diverses pathologies. L’hémochromatose génétique (i.e. absorption et stockage excessifs de fer dans l'organisme) ou une carence en fer (i.e. absorption et stockage insuffisants de fer) augmentent ainsi le risque de développer des maladies cardiovasculaires ou un diabète [7]. Des troubles de l'absorption, de l'excrétion et du stockage du fer contribuent également au développement de certains cancers comme le cancer du sein [8].

La quantité totale normale de fer dans l'organisme se situe en moyenne entre 3 et $4 \mathrm{~g}$ pour un individu de $70 \mathrm{~kg}$ [9]. Il est principalement présent dans l'hémoglobine des globules rouges représentant 65 à $70 \%$ du fer total de l'organisme. Le reste est essentiellement présent dans la myoglobine musculaire (i.e. 10 à $15 \%$ du fer total) et dans différents types d'organites cellulaires [10,11]. Le fer a la particularité d'être recyclé dans notre corps au niveau des macrophages majoritairement de la rate, pour être réincorporé principalement dans l'hème de l'hémoglobine lors de l'érythropoïèse au niveau de la moelle osseuse. Les pertes quotidiennes 
en fer sont extrêmement faibles en raison de l'absence de système actif d'excrétion ( $2 \mathrm{mg} / \mathrm{jour})$. Cette dernière est essentiellement passive via la desquamation des cellules cutanées et digestives, de la chute des cheveux, de la transpiration ou des pertes menstruelles chez la femme. Toutes ces excrétions sont compensées par le fer présent dans notre bol alimentaire dont l'absorption est finement contrôlée au niveau du tube digestif [12]. Ainsi, pour $10 \mathrm{mg}$ de fer apportés quotidiennement par un régime alimentaire équilibré, seulement 1 à $2 \mathrm{mg}$ sont absorbés au niveau intestinal. La Figure 1 schématise ainsi la distribution du fer dans l'organisme, alors que les tableaux 1 et 2 présentent respectivement les dosages classiquement utilisés dans un bilan martial et les apports journaliers recommandés en fer.

Le foie a un rôle essentiel dans la régulation du métabolisme du fer en synthétisant des protéines clés comme la transferrine, indispensable à son transport, ou la céruloplasmine essentielle à son oxydation dans le compartiment sanguin [10]. Le foie est également un organe de stockage majeur avec une capacité d'environ $1 \mathrm{~g}$, ce dernier pouvant accumuler le fer soit directement au sein des hépatocytes, ou bien au sein des macrophages hépatiques appelés également cellules de Kupffer. Le foie joue enfin un rôle essentiel dans l'homéostasie et la distribution du fer en synthétisant l'hepcidine, hormone clé considérée comme le chef d'orchestre du métabolisme du fer dans notre organisme.

Découverte au début des années 2000, l'hepcidine, est un peptide de 25 acides aminés riche en cystéine [13-15] permettant lorsqu'il est libéré par le foie de limiter l'absorption intestinale du fer, mais également de séquestrer ce dernier au sein des organes, essentiellement au niveau des macrophages de la rate. La ferroportine est actuellement considérée comme la seule protéine permettant aux cellules qui l'expriment d'exporter le fer qu'elles contiennent [16]. Le rôle de l'hepcidine est essentiel car il permet de limiter l'action de la ferroportine en conduisant à son internalisation et sa dégradation, ou en bloquant simplement l'action de la ferroportine [17]. Par ces mécanismes, l'hepcidine limite ainsi l'export du fer des entérocytes et des macrophages et conduit à réduire l'absorption intestinale et à séquestrer le fer dans les organes riches en macrophages [18](Figure 2).

L'hepcidine est au fer ce que l'insuline est au sucre, c'est une hormone dont la régulation est extrêmement sensible et qui répond à plusieurs signaux physiologiques afin de maintenir l'homéostasie du fer dans l'organisme [17]. Lorsque du fer se trouve en excès dans la circulation sanguine, les hépatocytes vont être les premières cellules dans lesquelles ce dernier va s'accumuler. Dans ce contexte, la protéine BMP6 (Bone Morphogenetic protein 6) est 
synthétisée par les cellules endothéliales du foie et relarguée dans le sang [19]. Elle se lie ensuite au complexe formé par son récepteur spécifique de la cellule hépatique appelé BMPr (BMP receptor) et à son co-récepteur l'hémojuvéline (HJV), ce qui permet la phosphorylation de facteurs de croissance de la famille des TGF $\beta$, les Smad1/5/8. Ces protéines forment ensuite un hétérotrimère avec Smad4 permettant sa translocation dans le noyau. Ce complexe transcriptionnel se lie alors sur le promoteur de transcription de l'hepcidine appelé BMP Response element (BMP-RE) [17](Figure 3). Par ailleurs, lorsque du fer se trouve en excès dans la circulation sanguine, la saturation en transferrine va augmenter conduisant à également stimuler la synthèse d'hepcidine. Les mécanismes sous-jacents de cette régulation, actuellement débattus, mettraient en jeu la protéine $\mathrm{HFE}$ et les récepteurs 1 et 2 de la transferrine (TFR1/TFR2). Plus spécifiquement, en cas de surcharge en fer systémique caractérisée par des concentrations élevées en holotransferrine (i.e. complexe fer-transferrine), HFE aurait tendance à se lier davantage à TFR2 ce qui aurait pour conséquence d'activer la voie ERK1/2 au sein des hépatocytes [20,21]. Cette activation permettrait alors de stimuler la transcription d'hepcidine [22] (Figure 3). Enfin, l'inflammation régule également positivement l'expression hépatique de l'hepcidine via la voie de signalisation JAK/STAT3. Cette voie est déclenchée par la sécrétion d'IL-6 au niveau circulant qui vient se fixer à son récepteur spécifique sur la membrane des hépatocytes (IL-6R). Cette interaction ligand-récepteur provoque la translocation nucléaire du facteur de transcription STAT3 capable de se lier au promoteur de l'hepcidine appelé GAS (interféron $\gamma$-activation sequence) [23](Figure 3).

\section{L'hypoactivité affecte à court terme l'homéostasie et la distribution du fer chez le rongeur}

Le modèle de suspension par la queue est actuellement le modèle de référence pour mimer les effets de l'hypoactivité chez le rongeur. Développé par la NASA dans les années 80, il permet de supprimer l'appui des pattes arrière et induit le déchargement du poids sur les pattes avant. Plus précisément, l'animal a les membres postérieurs en suspension formant un angle de $30^{\circ}$ entre le torse de l'animal et le sol. La fixation de la queue à un système de roulement permet son déplacement dans toute sa cage en limitant au maximum les contraintes mécaniques sur l'ensemble du corps [24]. Ce modèle permet ainsi d'observer une atrophie des muscles posturaux, une réduction de la densité minérale osseuse et une redistribution des fluides de l'organisme semblables à celles observées dans un organisme en impesanteur [24]. En s'appuyant sur ce modèle, nos équipes de recherche ont montré que 7 jours de suspension par la queue provoquaient une diminution des concentrations sériques en fer et de la saturation en 
transferrine [25]. Le fer circulant dans notre organisme dans un circuit fermé avec très peu de pertes, cette réduction si rapide de la biodisponibilité en fer ne pouvait s'expliquer que par une séquestration tissulaire. En poursuivant nos analyses, nous avons démontré que le fer s'accumulait effectivement dans la rate des animaux suspendus, alors que le foie demeurait préservé sur cette courte période d'hypoactivité [25]. Riche en macrophages, la rate constitue un organe essentiel pour dégrader les érythrocytes sénescents faisant de cette dernière un important lieu de stockage pour le fer [26,27].

Ce tableau clinique semblait typiquement indiquer une adaptation de l'organisme en situation d'inflammation. Pour vérifier cette hypothèse, nous nous sommes focalisés sur la régulation de l'hepcidine dans le foie de ces animaux. Pour la première fois, nous avons ainsi observé que les niveaux d'ARNm codant pour l'hepcidine étaient augmentés après une courte période d'hypoactivité, contribuant à expliquer en partie la séquestration du fer dans les macrophages spléniques. En étudiant les différentes voies de signalisation modulant sa transcription, nous avons également constatée que seule la voie IL-6/STAT3 était activée confirmant l'hypothèse d'une élévation de l'hepcidine d'origine pro-inflammatoire [25]. Ces résultats étaient d'ailleurs cohérents avec ceux de précédentes études ayant montré à la fois chez l'homme et le rongeur que l'hypoactivité augmentait à court terme les concentrations circulantes d'IL-6 [28-30]. En analysant les niveaux d'ARN codant pour IL-6 dans plusieurs organes, nous avons enfin observé que le seul organe présentant une élévation de ce paramètre était le muscle squelettique atrophié des animaux suspendus par la queue. L'ensemble de ces résultats démontraient ainsi chez le rongeur que l'hypoactivité redistribuait le fer dans l'organisme sous l'action de l'hepcidine, et suggéraient que l'atrophie musculaire pouvait jouer un rôle dans cette redistribution [25]. L'objectif de nos équipes fut alors 1) de déterminer si la redistribution du fer en hypoactivité était une réponse transitoire et adaptative ou bien pathologique, 2) de mieux cerner le rôle joué par le muscle squelettique dans cette redistribution du fer, et 3) de confirmer nos résultats chez l'homme.

\section{La distribution du fer dans l'organisme évolue selon la durée d'exposition à l'hypoactivité}

Afin de répondre en partie au premier objectif précédemment énoncé, nous avons relancé un protocole de suspension par la queue sur une période plus longue de 14 jours. Nous avons ainsi observé que la rate n'était plus surchargée en fer sur une période plus longue d'hypoactivité, malgré une réduction persistante de la biodisponibilité du fer [31]. Ces résultats suggèrent donc que le fer est toujours anormalement distribué à plus long terme, mais que les organes impactés 
ne sont pas nécessairement les mêmes. Le foie et le muscle squelettique atrophié ne seraient pas les cibles de cette surcharge au regard de l'absence de changements des concentrations en fer chez les animaux suspendus par la queue [31]. De manière intéressante, des travaux conduit parallèlement aux nôtres ont observé chez le rongeur que le foie et l'os commencent à être surchargés en fer après une plus longue période d'hypoactivité (i.e. 21 jours) [32]. Ensemble, ces résultats soulignent que la redistribution du fer se produisant en hypoactivité est dynamique dans le temps et affecte de manière différenciée nos organes. La séquestration de fer splénique se produisant à court terme pourrait ainsi constituer un mécanisme de protection préservant d'autres organes comme le foie et l'os d'une surcharge délétère en fer, ce mécanisme n'étant à priori plus efficace lorsque l'exposition à l'hypoactivité se prolonge.

Dans cette étude, nous avons également observé que les niveaux d'ARNm codant pour l'hepcidine restaient élevés dans le foie après 14 jours de suspension [31]. D'un côté, l'activation de la voie IL-6/STAT3 n'est plus visible suggérant que l'état pro-inflammatoire se réduit progressivement avec l'adaptation de l'organisme à son nouvel environnement. D'un autre côté, les voies BMP/SMAD et MAPK/ERK demeurent toujours inchangés comparativement aux animaux contrôles. Ensemble, ces données suggèrent que cette fenêtre temporelle de 14 jours ne serait finalement pas la meilleure pour étudier la régulation du métabolisme du fer chez le rongeur. Elle est probablement trop longue pour étudier les effets à court terme, et à l'inverse trop courte pour explorer les effets à long terme. En effet, après 21 jours de suspension, d'autres travaux commencent à observer une activation significative de la voie BMP/SMAD en parallèle d'une augmentation du contenu en fer hépatique [32]. En dépit de ces limites, l'ensemble de ces données supportent que 1) l'augmentation des niveaux d'ARNm codant pour l'hepcidine n'est pas une réponse transitoire à l'hypoactivité mais perdure dans le temps, et que 2) l'état pro-inflammatoire joue un rôle dans la régulation de l'hepcidine durant les premiers jours d'hypoactivité mais qu'ensuite, d'autres mécanismes prennent le relais.

\section{Quel rôle joue le muscle squelettique dans les altérations du métabolisme du fer en situation d'hypoactivité ?}

Dans notre étude précédente de 7 jours de suspension, nous avions émis l'hypothèse que l'atrophie musculaire induite par l'hypoactivité favorisait un état inflammatoire systémique stimulant par conséquent la synthèse hépatique d'hepcidine, responsable de la redistribution du fer au sein de l'organisme. Afin de vérifier expérimentalement cette hypothèse, nous avons par la suite relancé un protocole de 14 jours de suspension dans lequel une partie des rats étaient 
soumis à un reconditionnement intermittent, consistant à décrocher les animaux $1 \mathrm{~h} /$ jour pour limiter la perte de masse musculaire. Cependant, ce reconditionnement intermittent, pourtant efficace pour limiter l'atrophie musculaire, n'a induit aucun effet préventif sur le métabolisme du fer [31]. Cette absence totale d'effet sur le métabolisme du fer dans cette condition expérimentale réfute donc l'hypothèse d'un dialogue entre le muscle atrophié et le foie synthétisant l'hepcidine en situation d'hypoactivité. Toutefois, nous avons pris conscience que notre protocole présentait un certain nombre de limites et ne permettait pas de répondre à toutes les interrogations concernant un dialogue potentiel muscle-foie dans la régulation du métabolisme du fer. En effet, le reconditionnement intermittent que nous avons proposé s'est avéré efficace sur le soléaire, un muscle postural très impacté par la suspension. A l'inverse, aucun effet préventif ne fut observé sur le gastrocnémien, un muscle moins impacté par la suspension, mais qui constitue une masse musculaire environ 20 fois plus importante chez le rat. Ainsi, nous ne pouvons exclure que la contre-mesure proposée exerce un effet préventif trop limité sur l'ensemble de la masse musculaire atrophiée en réponse à l'hypoactivité pour agir sur la régulation du métabolisme du fer, et pour permettre de répondre à notre hypothèse initiale. Par ailleurs, nous avions émis l'hypothèse que le relargage musculaire d'IL-6 dans la circulation sanguine jouait un rôle dans la synthèse d'hepcidine. Or, après 14 jours de suspension, la voie IL-6/STAT3 n'est plus activée dans le foie des animaux suspendus, et ce, indépendamment du reconditionnement intermittent [31]. Nous ne pouvons donc exclure l'hypothèse que le reconditionnement intermittent exerce un effet préventif sur cette synthèse d'hepcidine dans les premiers jours d'hypoactivité, caractérisés par un état catabolique et inflammatoire intense au niveau musculaire. Cet effet s'estomperait alors au bout de 14 jours suivant la réduction progressive de l'état inflammatoire systémique et musculaire.

\section{Le métabolisme du fer est impacté en situation d'hypoactivité chez l'homme}

L'ensemble des résultats sur le métabolisme du fer obtenus chez le rongeur nous ont permis de participer en 2019 à une étude clinique conduite au sein de l'Institut de Médecine et de Physiologie Spatiales (MEDES, Toulouse). Cette étude consistait à exposer des hommes en bonne santé à 5 jours d'immersion sèche. Moins connu que le modèle d'alitement (i.e. bed rest), l'immersion sèche (en anglais dry immersion) commence à être de plus en plus utilisée pour mimer les effets de l'hypoactivité chez l'astronaute. Elle consiste à placer le sujet sur une membrane recouvrant une cuve d'eau, sur lequel le corps n'a pas d'appuis et est en état de 
flottaison induisant une hypoactivité sévère. Les altérations physiologiques sont ainsi observables beaucoup plus rapidement comparativement à l'alitement [33-35].

L'originalité de notre étude sur ce modèle d'immersion sèche était d'intégrer une méthode IRM non-invasive pour étudier le contenu en fer dans le foie et la rate des volontaires avant et après la période d'hypoactivité [36]. Grâce à cette approche, nous avons confirmé chez l'homme que le fer était séquestré dans la rate après quelques jours d'hypoactivité. Cette séquestration splénique était associée à une élévation des concentrations circulantes d'hepcidine confirmant le rôle clé de cette hormone dans la redistribution du fer en hypoactivité [37]. Mais contrairement aux rongeurs, nous avons constaté que le fer sérique et la saturation en transferrine augmentaient après 5 jours d'immersion sèche [37]. Malgré l'élévation des concentrations circulantes en hepcidine, ces résultats suggèrent donc chez l'homme que du fer s'accumule à court terme dans le compartiment sanguin en réponse à l'hypoactivité. L'enjeu est désormais de savoir si cet excès de fer dans la circulation sanguine perdure ou non lorsque l'exposition à l'hypoactivité se prolonge. Des valeurs chroniquement élevées exposeraient en effet l'organisme au risque de développer une surcharge en fer chronique dont l'impact sur la santé des astronautes ne serait pas négligeable.

Le métabolisme du fer fonctionnant en circuit fermé, nous nous sommes interrogés sur la provenance de ce fer s'accumulant dans le compartiment sanguin. Nos résultats suggèrent qu'une accélération de l'hémolyse et que l'atrophie musculaire jouerait un rôle important. Comme évoqué précédemment, 65 à $70 \%$ du fer de notre organisme est contenu dans l'hémoglobine des érythrocytes [38,39]. Il est bien admis depuis des décennies que l'hypoactivité est responsable d'une réduction de la masse totale d'hémoglobine caractérisant une anémie dont les mécanismes sont encore débattus [40,41]. Dans notre étude, nous avons observé que les concentrations en bilirubine non-conjuguée et en haptoglobine augmentaient à la suite de 5 jours d'immersion sèche [37]. Ces résultats suggèrent qu'une hémolyse extravasculaire pourrait s'opérer lors des premiers jours d'exposition à l'hypoactivité [42], et ainsi participer à relarguer du fer dans la circulation sanguine. L'atrophie musculaire pourrait également jouer un rôle important dans l'augmentation des concentrations sériques en fer et de la saturation en transferrine observé après 5 jours d'immersion sèche. En effet, le muscle squelettique contient 10 à $15 \%$ du fer sous forme de myoglobine. Comme de précédentes études $[34,43,44]$, nous avons observé une atrophie musculaire après 5 jours d'immersion sèche, associée à une élévation significative des concentrations circulantes de myoglobine. Tout 
comme l'hémoglobine, les macrophages sont capables de phagocyter la myoglobine pour dégrader ses groupements hème, et recycler le fer qu'ils contiennent [45]. Ainsi, nos données suggèrent que la perte de masse musculaire favoriserait un relargage de myoglobine dans le sang, qui après avoir été phagocytée par les macrophages spléniques pourrait contribuer à augmenter à la fois les concentrations circulantes et spléniques en fer.

\section{Conclusion et perspectives en termes de nutrition et d'activité physique}

L'ensemble des résultats que nous avons obtenu chez l'homme et le rongeur questionnent certains aspects de l'alimentation des astronautes exposés à la microgravité et l'hypoactivité dans la Station Spatiale Internationale (ISS). Alors que l'ANSES recommande des apports quotidiens en fer de entre 8 et $11 \mathrm{mg} / \mathrm{jour}$ pour l'homme, les apports dans l'alimentation des astronautes séjournant dans l'ISS sont en effet estimés à $20 \pm 6 \mathrm{mg} / \mathrm{jour}$ [46]. Ces valeurs peuvent même dépasser $35 \mathrm{mg} / \mathrm{jour}$ pour certains astronautes, principalement en raison de consommations de produits commerciaux fortifiés en fer [46]. Ainsi, nos résultats soulignent que ces consommations élevées en fer pourraient avoir des répercussions délétères sur l'organisme des astronautes présentant des anomalies de la distribution du fer. Ils suggèrent également l'importance de monitorer avec attention le statut martial des patients hospitalisés et alités de manière prolongée. Ces premières études ne permettent cependant pas encore de répondre à un certain nombre d'enjeux sanitaires à la fois pour les astronautes et les patients alités. Que ce soit chez le rongeur ou l'homme, nos résultats ont caractérisé le métabolisme du fer uniquement sur une période courte d'inactivité physique extrême (i.e. 5 à 14 jours). Nous ne pouvons exclure que les résultats obtenus caractérisent une réponse adaptative et transitoire de l'organisme à son nouvel environnement qui n'est pas nécessairement chronique et/ou pathologique sur le long terme. La durée des missions des astronautes pouvant s'étirer jusqu'à plusieurs années dans l'optique de se rendre sur Mars, il est important de déterminer si les anomalies du métabolisme du fer que nous avons constaté perdurent ou non, et si elles présentent un risque réel pour la santé de l'astronaute. Cet enjeu est de taille dans la mesure où l'organisme est également exposé dans l'espace aux rayonnements ionisants, reconnus pour accélérer les effets pro-oxydants du fer par la réaction de Fenton [47].

Par ailleurs, l'ensemble de nos données ont été collectées chez des rongeurs mâles ou des hommes, alors qu'il est admis que le métabolisme du fer est régulé différemment selon le sexe. En effet, en dehors des pertes sanguines liées aux menstruations chez la femme, le sexe module de façon importante le métabolisme du fer comme cela est illustré au cours de 
l'hémochromatose génétique, archétype des pathologies de surcharge en fer [48,49]. Alors que la prédisposition génétique à l'hémochromatose génétique (homozygotie C282Y) est similaire en fréquence chez les hommes et les femmes, ces dernières présentent des symptômes moins graves de la pathologie, puisqu'elles accumulent moins fréquemment et rapidement du fer, et présentent une plus faible incidence des complications [48-50]. Nous émettons donc l'hypothèse que l'adaptation du métabolisme du fer à l'hypoactivité pourrait varier selon le sexe. De telles différences pourraient avoir un impact sur les anomalies du métabolisme du fer observées, et donc sur les recommandations, notamment nutritionnelles ou pharmacologiques faites aux astronautes. Ces différences pourraient également influencer leur sensibilité aux rayonnements ionisants particulièrement élevés dans l'espace [47].

Caractériser l'impact à court et long terme de l'inactivité physique extrême sur le métabolisme du fer chez l'homme comme la femme apparait indispensable pour améliorer et individualiser au mieux la prise en charge des astronautes, mais également des patients alités quel qu'en soit la raison. Mais l'efficacité ou les risques associées à certaines stratégies ou contremesures peuvent être d'ores et déjà et parallèlement évaluées. Même si ces modalités demandent à être encore optimisées, l'exercice physique est une contremesure dont l'efficacité pour limiter l'atrophie musculaire et l'ostéoporose chez les astronautes a été démontrée [51-53]. Toutefois, les répercussions de ces séances d'exercice physique quotidiennes sur le métabolisme du fer de l'astronaute ne sont pas encore identifiées. Sur Terre, il est admis qu'un exercice physique qu'il soit d'intensité modérée ou intense induit une élévation des niveaux circulants d'hepcidine [5456], principalement en stimulant la voie pro-inflammatoire IL-6/STAT3 [57]. Toutefois, l'amplitude de cette réponse semble dépendante du statut martial et inflammatoire de l'individu $[58,59]$. Nos travaux ont souligné que l'hypoactivité augmentait de manière chronique les niveaux circulants d'hepcidine et induisait une redistribution anormale du fer. Dans ce contexte, il apparait donc légitime de se questionner sur l'impact à court et long terme de l'exercice physique sur le métabolisme du fer dans ce contexte d'hypoactivité, et d'étudier les répercussions bénéfiques ou délétères de ses effets sur la santé de l'astronaute.

\section{Remerciements}

Ce travail a reçu le soutien financier du Centre National d'Etudes Spatiales (CNES), de la région Bretagne et de l'Institut national de recherche pour l'agriculture, l'alimentation et l'environnement (INRAE). 


\section{Déclaration de liens d'intérêt}

Les auteurs déclarent ne pas avoir de liens d'intérêts.

\section{Références}

[1] Grimm D, Grosse J, Wehland M, Mann V, Reseland JE, Sundaresan A, et al. The impact of microgravity on bone in humans. Bone 2016;87:44-56. https://doi.org/10.1016/j.bone.2015.12.057.

[2] Mulder E, Clément G, Linnarsson D, Paloski WH, Wuyts FP, Zange J, et al. Musculoskeletal effects of 5 days of bed rest with and without locomotion replacement training. Eur J Appl Physiol 2015;115:727-38. https://doi.org/10.1007/s00421-014-30450 .

[3] Greaves D, Arbeille P, Guillon L, Zuj K, Caiani EG. Effects of exercise countermeasure on myocardial contractility measured by 4D speckle tracking during a 21-day head-down bed rest. Eur J Appl Physiol 2019;119:2477-86. https://doi.org/10.1007/s00421-01904228-0.

[4] Coker RH, Hays NP, Williams RH, Wolfe RR, Evans WJ. Bed Rest Promotes Reductions in Walking Speed, Functional Parameters, and Aerobic Fitness in Older, Healthy Adults. The Journals of Gerontology Series A: Biological Sciences and Medical Sciences 2015;70:91-6. https://doi.org/10.1093/gerona/glu123.

[5] Moore AD, Downs ME, Lee SMC, Feiveson AH, Knudsen P, Ploutz-Snyder L. Peak exercise oxygen uptake during and following long-duration spaceflight. Journal of Applied Physiology 2014;117:231-8. https://doi.org/10.1152/japplphysiol.01251.2013.

[6] Aisen P, Enns C, Wessling-Resnick M. Chemistry and biology of eukaryotic iron metabolism. Int J Biochem Cell Biol 2001;33:940-59.

[7] Liu Q, Sun L, Tan Y, Wang G, Lin X, Cai L. Role of iron deficiency and overload in the pathogenesis of diabetes and diabetic complications. Curr Med Chem 2009;16:113-29.

[8] Marques O, da Silva BM, Porto G, Lopes C. Iron homeostasis in breast cancer. Cancer Lett 2014;347:1-14. https://doi.org/10.1016/j.canlet.2014.01.029.

[9] Ganz T. Iron Metabolism. In: Kaushansky K, Lichtman MA, Prchal JT, Levi MM, Press OW, Burns LJ, et al., editors. Williams Hematology. 9th ed., New York, NY: McGrawHill Education; 2015.

[10] Brissot P, Loréal O. Iron metabolism and related genetic diseases: A cleared land, keeping mysteries. J Hepatol 2016;64:505-15. https://doi.org/10.1016/j.jhep.2015.11.009.

[11] Anderson GJ, Frazer DM. Current understanding of iron homeostasis. Am J Clin Nutr 2017;106:1559S-1566S. https://doi.org/10.3945/ajen.117.155804. 
[12] Loréal O, Cavey T, Bardou-Jacquet E, Guggenbuhl P, Ropert M, Brissot P. Iron, hepcidin, and the metal connection. Front Pharmacol 2014;5:128. https://doi.org/10.3389/fphar.2014.00128.

[13] Nicolas G, Bennoun M, Devaux I, Beaumont C, Grandchamp B, Kahn A, et al. Lack of hepcidin gene expression and severe tissue iron overload in upstream stimulatory factor 2 (USF2) knockout mice. Proc Natl Acad Sci USA 2001;98:8780-5. https://doi.org/10.1073/pnas.151179498.

[14] Park CH, Valore EV, Waring AJ, Ganz T. Hepcidin, a urinary antimicrobial peptide synthesized in the liver. J Biol Chem 2001;276:7806-10. https://doi.org/10.1074/jbc.M008922200.

[15] Pigeon C, Ilyin G, Courselaud B, Leroyer P, Turlin B, Brissot P, et al. A new mouse liverspecific gene, encoding a protein homologous to human antimicrobial peptide hepcidin, is overexpressed during iron overload. J Biol Chem 2001;276:7811-9. https://doi.org/10.1074/jbc.M008923200.

[16] Ganz T. Systemic iron homeostasis. Physiol Rev 2013;93:1721-41. https://doi.org/10.1152/physrev.00008.2013.

[17] Aschemeyer S, Qiao B, Stefanova D, Valore EV, Sek AC, Ruwe TA, et al. Structurefunction analysis of ferroportin defines the binding site and an alternative mechanism of action of hepcidin. Blood 2018;131:899-910. https://doi.org/10.1182/blood-2017-05786590.

[18] Zhang A-S. Control of Systemic Iron Homeostasis by the Hemojuvelin-Hepcidin Axis12. Adv Nutr 2010;1:38-45. https://doi.org/10.3945/an.110.1009.

[19] Canali S, Zumbrennen-Bullough KB, Core AB, Wang C-Y, Nairz M, Bouley R, et al. Endothelial cells produce bone morphogenetic protein 6 required for iron homeostasis in mice. Blood 2017;129:405-14. https://doi.org/10.1182/blood-2016-06-721571.

[20] Bayele HK, Srai SKS. Genetic variation in hepcidin expression and its implications for phenotypic differences in iron metabolism. Haematologica 2009;94:1185-8. https://doi.org/10.3324/haematol.2009.010793.

[21] Goswami T, Andrews NC. Hereditary hemochromatosis protein, HFE, interaction with transferrin receptor 2 suggests a molecular mechanism for mammalian iron sensing. J Biol Chem 2006;281:28494-8. https://doi.org/10.1074/jbc.C600197200.

[22] Lawen A, Lane DJR. Mammalian iron homeostasis in health and disease: uptake, storage, transport, and molecular mechanisms of action. Antioxid Redox Signal 2013;18:2473507. https://doi.org/10.1089/ars.2011.4271.

[23] Wrighting DM, Andrews NC. Interleukin-6 induces hepcidin expression through STAT3. Blood 2006;108:3204-9. https://doi.org/10.1182/blood-2006-06-027631.

[24] Globus RK, Morey-Holton E. Hindlimb unloading: rodent analog for microgravity. J Appl Physiol 2016;120:1196-206. https://doi.org/10.1152/japplphysiol.00997.2015. 
[25] Cavey T, Pierre N, Nay K, Allain C, Ropert M, Loréal O, et al. Simulated microgravity decreases circulating iron in rats: role of inflammation-induced hepcidin upregulation. Exp Physiol 2017;102:291-8. https://doi.org/10.1113/EP086188.

[26] Korolnek T, Hamza I. Macrophages and iron trafficking at the birth and death of red cells. Blood 2015;125:2893-7. https://doi.org/10.1182/blood-2014-12-567776.

[27] Soares MP, Hamza I. Macrophages and iron metabolism. Immunity 2016;44:492-504. https://doi.org/10.1016/j.immuni.2016.02.016.

[28] Bosutti A, Malaponte G, Zanetti M, Castellino P, Heer M, Guarnieri G, et al. Calorie restriction modulates inactivity-induced changes in the inflammatory markers C-reactive protein and pentraxin-3. J Clin Endocrinol Metab 2008;93:3226-9. https://doi.org/10.1210/jc.2007-1684.

[29] Felix K, Wise K, Manna S, Yamauchi K, Wilson BL, Thomas RL, et al. Altered cytokine expression in tissues of mice subjected to simulated microgravity. Mol Cell Biochem 2004;266:79-85.

[30] Yakabe M, Ogawa S, Ota H, Iijima K, Eto M, Ouchi Y, et al. Inhibition of interleukin-6 decreases atrogene expression and ameliorates tail suspension-induced skeletal muscle atrophy. PLoS One 2018;13. https://doi.org/10.1371/journal.pone.0191318.

[31] Nay K, Martin D, Orfila L, Saligaut D, Martin B, Horeau M, et al. Intermittent reloading does not prevent iron availability decrease and hepcidin upregulation caused by hindlimb unloading. Exp Physiol 2020. https://doi.org/10.1113/EP088339.

[32] Xu Z, Sun W, Li Y, Ling S, Zhao C, Zhong G, et al. The regulation of iron metabolism by hepcidin contributes to unloading-induced bone loss. Bone 2017;94:152-61. https://doi.org/10.1016/j.bone.2016.09.023.

[33] De Abreu S, Amirova L, Murphy R, Wallace R, Twomey L, Gauquelin-Koch G, et al. Multi-System Deconditioning in 3-Day Dry Immersion without Daily Raise. Front Physiol 2017;8:799. https://doi.org/10.3389/fphys.2017.00799.

[34] Demangel R, Treffel L, Py G, Brioche T, Pagano AF, Bareille M-P, et al. Early structural and functional signature of 3-day human skeletal muscle disuse using the dry immersion model. J Physiol (Lond) 2017;595:4301-15. https://doi.org/10.1113/JP273895.

[35] Navasiolava NM, Custaud M-A, Tomilovskaya ES, Larina IM, Mano T, Gauquelin-Koch G, et al. Long-term dry immersion: review and prospects. Eur J Appl Physiol 2011;111:1235-60. https://doi.org/10.1007/s00421-010-1750-x.

[36] Gandon Y, Olivié D, Guyader D, Aubé C, Oberti F, Sebille V, et al. Non-invasive assessment of hepatic iron stores by MRI. Lancet 2004;363:357-62. https://doi.org/10.1016/S0140-6736(04)15436-6.

[37] Nay K, Koechlin-Ramonatxo C, Rochdi S, Island M-L, Orfila L, Treffel L, et al. Simulated microgravity disturbs iron metabolism and distribution in humans: Lessons from dry 
immersion, an innovative ground-based human model. The FASEB Journal 2020;[Epub ahead of print]. https://doi.org/10.1096/fj.202001199RR.

[38] Andrews NC. Disorders of iron metabolism. N Engl J Med 1999;341:1986-95. https://doi.org/10.1056/NEJM199912233412607.

[39] Muñoz M, García-Erce JA, Remacha AF. Disorders of iron metabolism. Part 1: molecular basis of iron homoeostasis. J Clin Pathol 2011;64:281-6. https://doi.org/10.1136/jcp.2010.079046.

[40] De Santo NG, Cirillo M, Kirsch KA, Correale G, Drummer C, Frassl W, et al. Anemia and erythropoietin in space flights. Semin Nephrol 2005;25:379-87. https://doi.org/10.1016/j.semnephrol.2005.05.006.

[41] Smith SM. Red blood cell and iron metabolism during space flight. Nutrition 2002;18:864-6.

[42] Mebius RE, Kraal G. Structure and function of the spleen. Nat Rev Immunol 2005;5:60616. https://doi.org/10.1038/nri1669.

[43] Fitts RH, Riley DR, Widrick JJ. Functional and structural adaptations of skeletal muscle to microgravity. J Exp Biol 2001;204:3201-8.

[44] Dirks ML, Smeets JSJ, Holwerda AM, Kouw IWK, Marzuca-Nassr GN, Gijsen AP, et al. Dietary feeding pattern does not modulate the loss of muscle mass or the decline in metabolic health during short-term bed rest. Am J Physiol Endocrinol Metab 2019;316:E536-45. https://doi.org/10.1152/ajpendo.00378.2018.

[45] Corna G, Caserta I, Monno A, Apostoli P, Manfredi AA, Camaschella C, et al. The Repair of Skeletal Muscle Requires Iron Recycling through Macrophage Ferroportin. J Immunol 2016;197:1914-25. https://doi.org/10.4049/jimmunol.1501417.

[46] Smith SM, Rice BL, Dlouhy H, Zwart SR. Assessment of Nutritional Intake During Space Flight and Space Flight Analogs. Procedia Food Science 2013;2:27-34. https://doi.org/10.1016/j.profoo.2013.04.006.

[47] Yang J, Zhang G, Dong D, Shang P. Effects of Iron Overload and Oxidative Damage on the Musculoskeletal System in the Space Environment: Data from Spaceflights and Ground-Based Simulation Models. Int J Mol Sci 2018;19. https://doi.org/10.3390/ijms19092608.

[48] Allen KJ, Gurrin LC, Constantine CC, Osborne NJ, Delatycki MB, Nicoll AJ, et al. Ironoverload-related disease in HFE hereditary hemochromatosis. $N$ Engl $J$ Med 2008;358:221-30. https://doi.org/10.1056/NEJMoa073286.

[49] Harrison-Findik DD. Gender-related variations in iron metabolism and liver diseases. World J Hepatol 2010;2:302-10. https://doi.org/10.4254/wjh.v2.i8.302. 
[50] Moirand R, Adams PC, Bicheler V, Brissot P, Deugnier Y. Clinical features of genetic hemochromatosis in women compared with men. Ann Intern Med 1997;127:105-10. https://doi.org/10.7326/0003-4819-127-2-199707150-00002.

[51] Hargens AR, Bhattacharya R, Schneider SM. Space physiology VI: exercise, artificial gravity, and countermeasure development for prolonged space flight. Eur J Appl Physiol 2013;113:2183-92. https://doi.org/10.1007/s00421-012-2523-5.

[52] Jones TW, Petersen N, Howatson G. Optimization of Exercise Countermeasures for Human Space Flight: Operational Considerations for Concurrent Strength and Aerobic Training. Front Physiol 2019;10:584. https://doi.org/10.3389/fphys.2019.00584.

[53] Laurens C, Simon C, Vernikos J, Gauquelin-Koch G, Blanc S, Bergouignan A. Revisiting the Role of Exercise Countermeasure on the Regulation of Energy Balance During Space Flight. Front Physiol 2019;10:321. https://doi.org/10.3389/fphys.2019.00321.

[54] Peeling P. Exercise as a mediator of hepcidin activity in athletes. Eur J Appl Physiol 2010;110:877-83. https://doi.org/10.1007/s00421-010-1594-4.

[55] Sim M, Garvican-Lewis LA, Cox GR, Govus A, McKay AKA, Stellingwerff T, et al. Iron considerations for the athlete: a narrative review. Eur J Appl Physiol 2019;119:1463-78. https://doi.org/10.1007/s00421-019-04157-y.

[56] Troadec M-B, Lainé F, Daniel V, Rochcongar P, Ropert M, Cabillic F, et al. Daily regulation of serum and urinary hepcidin is not influenced by submaximal cycling exercise in humans with normal iron metabolism. Eur J Appl Physiol 2009;106:435-43. https://doi.org/10.1007/s00421-009-1031-8.

[57] Banzet S, Sanchez H, Chapot R, Bigard X, Vaulont S, Koulmann N. Interleukin-6 contributes to hepcidin mRNA increase in response to exercise. Cytokine 2012;58:15861. https://doi.org/10.1016/j.cyto.2012.01.006.

[58] Domínguez R, Sánchez-Oliver AJ, Mata-Ordoñez F, Feria-Madueño A, Grimaldi-Puyana M, López-Samanes Á, et al. Effects of an Acute Exercise Bout on Serum Hepcidin Levels. Nutrients 2018;10. https://doi.org/10.3390/nu10020209.

[59] Peeling P, Sim M, Badenhorst CE, Dawson B, Govus AD, Abbiss CR, et al. Iron status and the acute post-exercise hepcidin response in athletes. PLoS ONE 2014;9:e93002. https://doi.org/10.1371/journal.pone.0093002. 


\section{Tableaux et légendes}

\begin{tabular}{|c|c|c|c|c|c|}
\hline Paramètres sanguins & Valeurs normales & $\begin{array}{l}\text { Carence en fer } \\
\text { sans anémie }\end{array}$ & $\begin{array}{l}\text { Anémie } \\
\text { ferriprive }\end{array}$ & $\begin{array}{c}\text { Anémie } \\
\text { inflammatoire }\end{array}$ & Surcharge en fer \\
\hline Hémoglobine & $\begin{array}{l}\mathrm{H}=13.5-17.5 \mathrm{~g} / \mathrm{dl} \\
\mathrm{F}=12.5-15.5 \mathrm{~g} / \mathrm{dl}\end{array}$ & $=$ & $y$ & $y$ & $=$ \\
\hline Fer sérique & $\begin{array}{c}\mathrm{H}=10-30 \mu \mathrm{mol} / \mathrm{L} \\
\mathrm{F}=9-28 \mu \mathrm{mol} / \mathrm{L}\end{array}$ & $\mathbf{y}$ & $\mathbf{y}$ & $\mathbf{y}$ & $\pi$ \\
\hline Saturation en transferrine & $20-40 \%$ & $y$ & $y$ & y & $\pi$ \\
\hline Ferritine & $\begin{array}{l}\mathrm{H}=20-250 \mu \mathrm{g} / \mathrm{L} \\
\mathrm{F}=15-150 \mu \mathrm{g} / \mathrm{L}\end{array}$ & $\mathbf{y}$ & $\mathbf{y}$ & $=$ ou $\pi$ & $\pi$ \\
\hline Protéine C Réactive (CRP) & $<6 \mathrm{mg} / \mathrm{L}$ & $=$ & $=$ & $\pi$ & $=$ \\
\hline $\begin{array}{l}\text { Récepteur soluble de la } \\
\text { transferrine }\end{array}$ & $0.76-1.76 \mathrm{mg} / \mathrm{L}$ & $\pi$ & $\pi$ & $=$ & $y$ \\
\hline
\end{tabular}

Tableau 1. Valeurs de référence des principaux paramètres étudiés lors d'un bilan martial et tableaux cliniques associés aux principaux désordres du métabolisme du fer. $\mathrm{H}$ : homme, $\mathrm{F}$ : femme

\begin{tabular}{|ccccc}
\hline Age & Homme & Femme & Grossesse & Allaitement \\
\hline Naissance à $\mathbf{6}$ mois & $0.27 \mathrm{mg}$ & $0.27 \mathrm{mg}$ & & \\
$\mathbf{7}$ à $\mathbf{1 2}$ mois & $11 \mathrm{mg}$ & $11 \mathrm{mg}$ & \\
$\mathbf{1}$ à $\mathbf{3}$ ans & $7 \mathrm{mg}$ & $7 \mathrm{mg}$ & \\
$\mathbf{4}$ à $\mathbf{8}$ ans & $10 \mathrm{mg}$ & $10 \mathrm{mg}$ & \\
$\mathbf{9}$ à $\mathbf{1 3}$ ans & $8 \mathrm{mg}$ & $8 \mathrm{mg}$ & $27 \mathrm{mg}$ & $9 \mathrm{mg}$ \\
$\mathbf{1 4}$ à $\mathbf{1 8}$ ans & $11 \mathrm{mg}$ & $15 \mathrm{mg}$ & $27 \mathrm{mg}$ & \\
$\mathbf{1 9}$ à $\mathbf{5 0}$ ans & $8 \mathrm{mg}$ & $18 \mathrm{mg}$ & & \\
$+\mathbf{d e} \mathbf{5 0}$ ans & $8 \mathrm{mg}$ & $8 \mathrm{mg}$ & &
\end{tabular}

Tableau 2. Apports Journaliers Recommandés (AJR) en fer [9] 


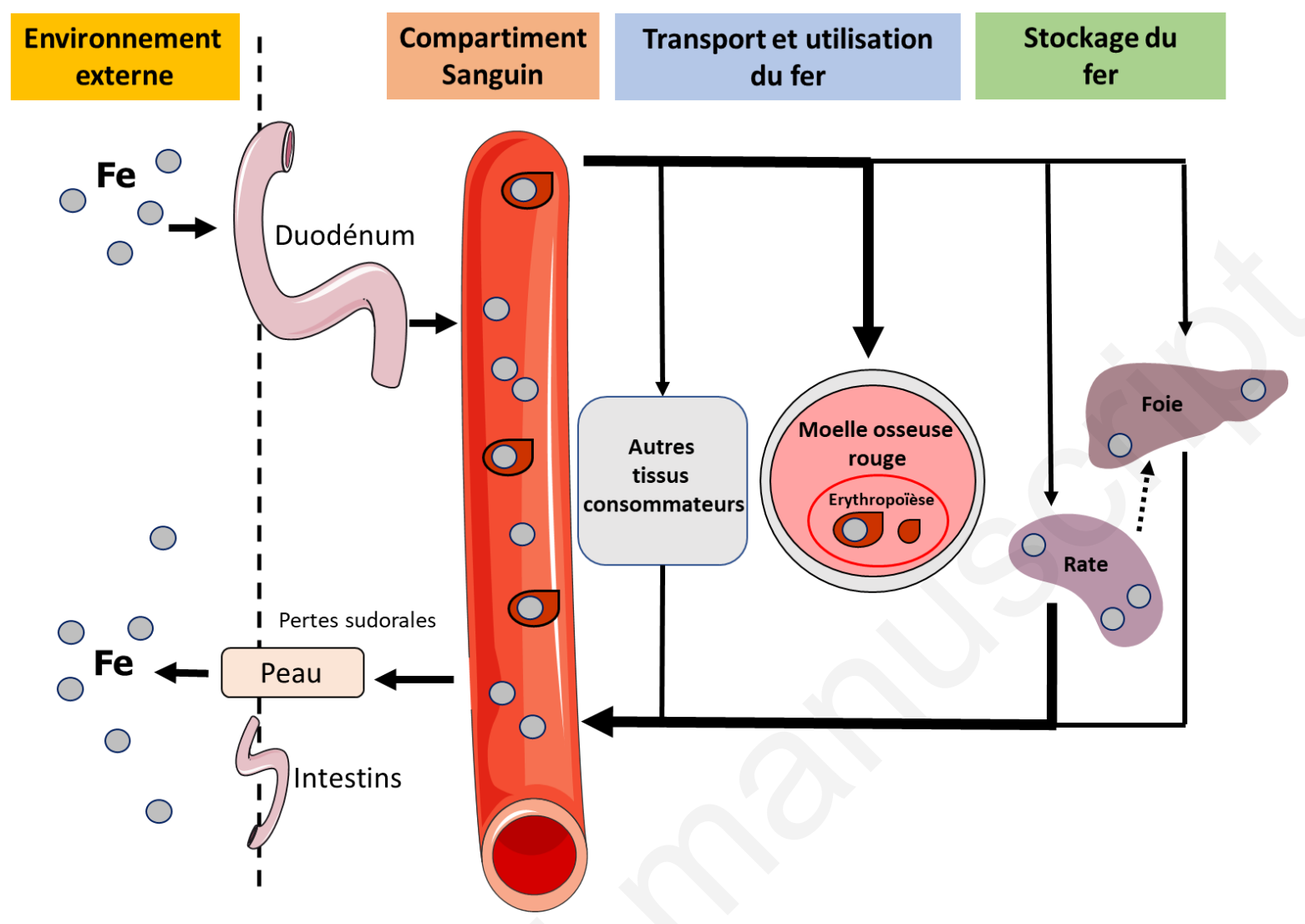

Figure 1. Distribution du fer dans l'organisme. Le fer est absorbé au niveau de l'intestin grêle et plus particulièrement du duodénum grâce aux cellules des villosités de ce dernier, les entérocytes. Les cellules se servent du fer absorbé pour synthétiser de nombreuses protéines et organites utilisés dans diverses réactions cellulaires. Le fer est toutefois principalement utilisé lors de l'érythropoïèse dans la moelle osseuse rouge pour se lier au cofacteur hème dans les globules rouges. Le fer présent dans 1'hémoglobine des globules rouges représente 65 à $70 \%$ du fer total de l'organisme. Lorsque le fer n'est pas utilisé pour les besoins cellulaires, il est stocké sous forme de ferritine dans les organes. Pour assurer les besoins en fer de l'organisme, les érythrocytes sénescents sont dégradés par les macrophages tissulaires principalement au niveau de la rate. Ce processus est appelé érythrophagocytose. Les pertes de fer sont très faibles et passives via la desquamation des cellules cutanées et digestives, de la chute des cheveux, la transpiration ou les pertes menstruelles chez la femme. 


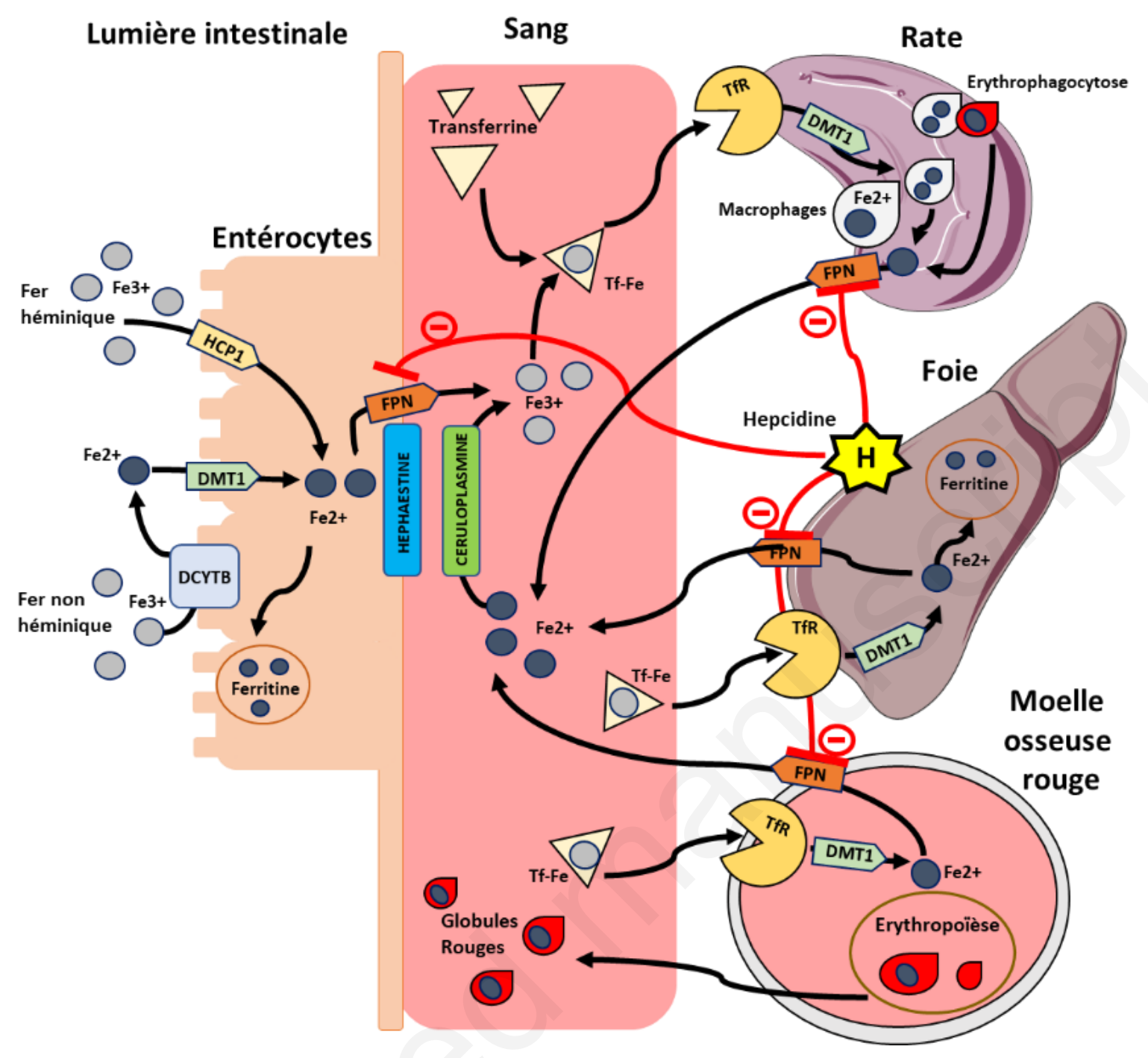

Figure 2. L'hepcidine : chef d'orchestre du métabolisme du fer. Le fer ferrique libre présent dans les intestins $\left(\mathrm{Fe}^{3+}\right)$ est réduit en $\mathrm{Fe}^{2+}$ par le duodenal cytochrome b (DCYTB) en entrant dans la cellule de l'entérocyte via le transporteur DMT1. L'hème alimentaire est directement absorbé et le fer est relargué grâce à l'heam carrier protein 1 (HCP1). Le fer quitte l'entérocyte via la ferroportine (FPN) pour se retrouver dans la circulation après avoir subi une oxydation par l'hépheastine pour se lier à la transferrine (Tf). Ce complexe $\mathrm{Tf}-\mathrm{Fe}^{3+}$ se lie à ses récepteurs TfR1 et TfR2 sur les cellules cibles. Après l'endocytose de TfR1 et l'acidification de l'endosome, $\mathrm{Fe}^{3+}$ est réduit par la métalloréductase STEAP et transporté par DMT1 dans le cytosol afin d'être utilisé ou séquestré sous forme de ferritine. La concentration sanguine en ferritine est ainsi un marqueur de stockage du fer par les organes. Le complexe Tf-Fe peut aussi se fixer sur TfR2 du foie où il sera stocké et activera des voies de signalisations nécessaires à la sécrétion d'hepcidine $(\mathrm{H})$ qui inhibera à son tour l'expression et l'action de la FPN, ce qui limitera l'absorption du fer par rétrocontrôle négatif. 


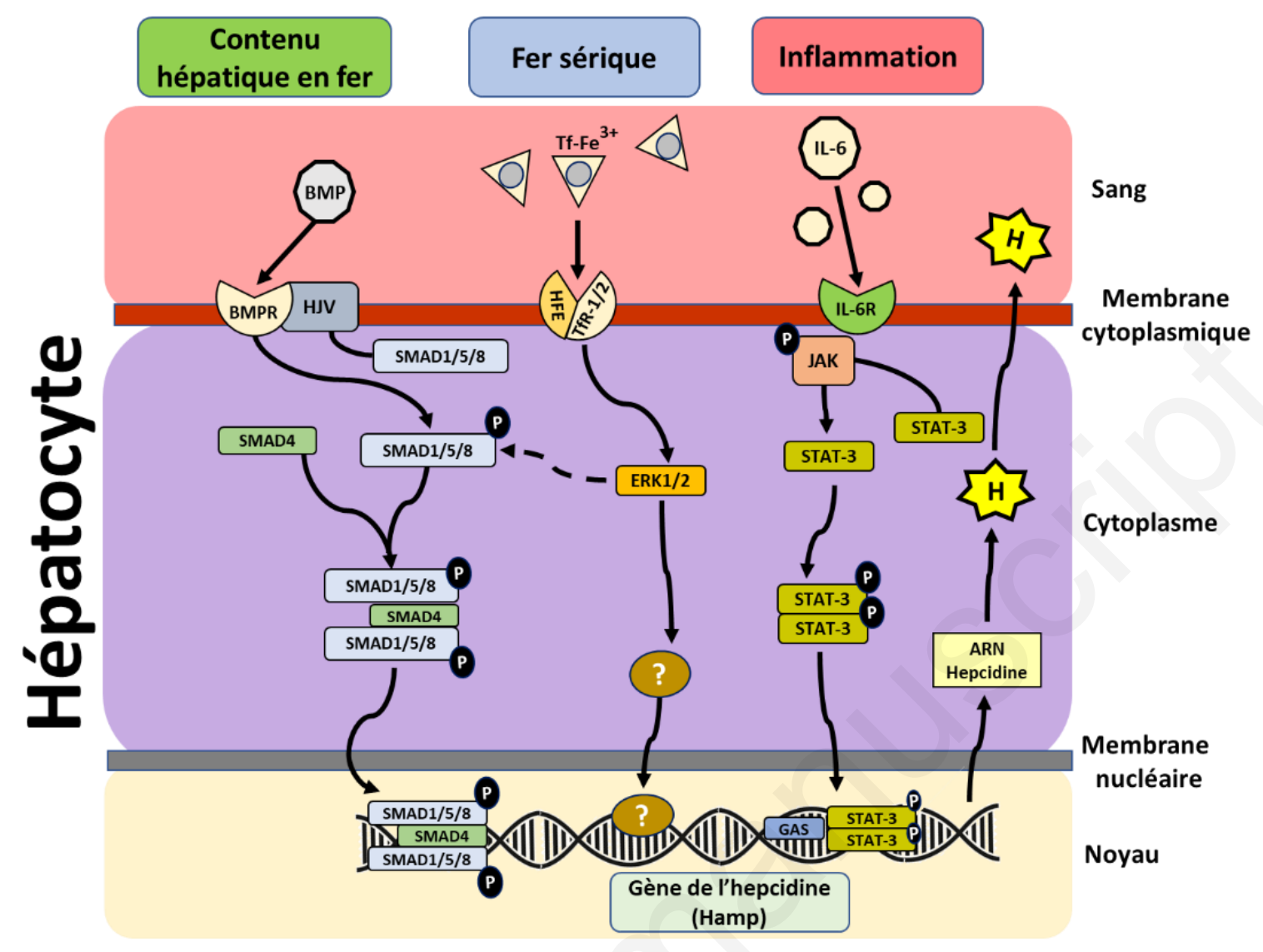

Figure 3. Mécanismes stimulant la synthèse d'hepcidine dans le foie.

Voie BMP/SMAD (simplifiée) : Lorsque les concentrations hépatiques en fer sont trop importantes, la protéine BMP6 (Bone Morphogenetic protein 6) est synthétisée par les cellules endothéliales du foie et relarguée dans le sang. Elle se lie alors au complexe formé par son récepteur spécifique des hépatocytes appelé BMPr (BMP receptor) et à son co-récepteur l'hémojuvéline (HJV), ce qui permet la phosphorylation de facteurs de croissance de la famille des TGF $\beta$, les Smad1/5/8. Ces protéines forment ensuite un hétérotrimère avec Smad4 permettant sa translocation dans le noyau. Ce complexe transcriptionnel se lie alors sur un promoteur de l'hepcidine appelé BMP Response element (BMP-RE) Voie MAPK/ERK : Ce mécanisme, encore objet de débat, mettrait en jeu la protéine HFE et les récepteurs 1 (TFR1) et 2 de la transferrine (TFR2). En condition normale, la protéine HFE entre en compétition avec la transferrine pour se fixer à TFR1 en raison d'un site de fixation qui chevaucherait celui de la transferrine. A l'inverse, TFR2 est capable de se lier en même temps à HFE et la transferrine. Ainsi, en cas de surcharge en fer systémique caractérisée par des concentrations élevées en complexe fer-transferrine, HFE aurait tendance à se lier davantage à TFR2 ce qui aurait pour conséquence d'activer la voie ERK1/2 (Goswami and Andrews, 2006). Cette activation permet 
alors de stimuler un promoteur de l'hepcidine appelée HAMP. De plus, ERK1 et ERK2 moduleraient positivement la phosphorylation de SMAD participant ainsi à l'activation de la voie BMP/SMAD Inflammation - Voie JAK/STAT3 : Cette voie est déclenchée par la sécrétion d'IL-6 au niveau circulant qui vient se fixer à son récepteur spécifique sur la membrane des hépatocytes (IL-6R). Cette interaction ligand-récepteur cause la phosphorylation de Janus kinase (JAK) dans le cytosol conduisant à son tour à une phosphorylation du facteur de transcription STAT3. Une fois phosphorylées, deux protéines STAT3 forment un homodimère qui se transloque dans le noyau et se lie au promoteur de l'hepcidine appelé GAS (l'interféron $\gamma$-activation sequence). 